\title{
Language development in Japanese children who receive cochlear implant and/or hearing aid
}

Running title: language development in pediatric cochlear implantation

Satoshi Iwasaki ${ }^{1)}$, Shinya Nishio ${ }^{2)}$, Hideaki Moteki ${ }^{1,2)}$, Yutaka Takumi ${ }^{1,2)}$, Kunihiro Fukushima ${ }^{3),}$ Norio Kasai ${ }^{3,4)}$, Shin-ichi Usami ${ }^{2)}$

${ }^{1)}$ Department of Hearing Implant Sciences, Shinshu University School of Medicine; ${ }^{2}$ Department of Otolaryngology, Shinshu University School of Medicine; ${ }^{3)}$ Department of Otolaryngology, Head and Neck Surgery, and Okayama University Postgraduate School of Medicine, Dentistry, and Pharmaceutical Science; ${ }^{4}$ The Association for Technical Aids

Key words: Congenital hearing loss, language development, cochlear implant, communication

Correspondence to: Satoshi Iwasaki, MD 
Department of Hearing Implant Sciences, Shinshu University School of Medicine, 3-1-1 Asahi, Matsumoto 390-8621, Japan

Tel: +81-263-37-2666

Fax: $+81-263-36-9164$

E-mail: iwasakis@shinshu-u.ac.jp 


\section{Abstract}

Objectives: This study aimed to investigate a wide variety of factors that influence auditory, speech, and language development following pediatric cochlear implantation (CI).

Study design: Prospective collection of language tested data in profound hearing-impaired children.

Hypothesis: Pediatric CI can potentially be effective to development of practical communication skills and early implantation is more effective.

Methods: We proposed a set of language tests (assessment package of the language development for Japanese hearing-impaired children; ALADJIN) consisting of communication skills testing (test for question-answer interaction development; TQAID), comprehensive (Peabody Picture Vocabulary Test-Revised; PVT-R and Standardized Comprehension Test for Abstract Words; SCTAW) and productive vocabulary (Word Fluency Test; WFT), and comprehensive and productive syntax (Syntactic processing Test for Aphasia; STA). Of 638 hearing-impaired children recruited for this study, $282(44.2 \%)$ with $>70 \mathrm{~dB}$ hearing impairment had undergone CI. After excluding children with low birth weight $(<1800 \mathrm{~g})$, those with $>11$ points on 
the Pervasive Developmental Disorder ASJ Rating Scale for the test of autistic tendency, and those $<2 \mathrm{SD}$ on Raven's Colored Progressive Matrices for the test of non-verbal intelligence, 190 children were subjected to this set of language tests.

Results: Sixty children (31.6\%) were unilateral CI-only users, 128 (67.4\%) were CI-hearing aid (HA) users, and 2 (1.1\%) were bilateral CI users. Hearing loss level of CI users was significantly $(\mathrm{p}<0.01)$ worse than that of HA-only users. However, the threshold level, maximum speech discrimination score, and speech intelligibility rating in CI users were significantly $(\mathrm{p}<0.01)$ better than those in HA-only users. The scores for PVT-R $(\mathrm{p}<0.01)$, SCTAW, and WFT in CI users were better than those in HA-only users. STA and TQAID scores in CI-HA users were significantly $(p<0.05)$ better than those in unilateral CI-only users. The high correlation $(r=0.52)$ has been found between the age of CI and maximum speech discrimination score. The scores of speech and language tests in the implanted children before 24 months of age have been better than those in the implanted children after 24 months of age.

Conclusions: We could indicate that CI was effective for language 
development in Japanese hearing-impaired children and early CI was more effective for productive vocabulary and syntax. 


\section{Introduction}

Management of $\mathrm{CI}$ in infants and children is one of the most striking advances for congenital severe to profound hearing loss. Several studies have shown that early implantation can be beneficial not only for speech perception, but also for the development of speech and language (1-3). Moreover, early intervention for children with hearing loss facilitates successful educational integration at the earliest possible age (4).

More than 20 years have passed since the first pediatric CI surgery was performed in Japan. Many hearing-impaired children are now benefiting from this device. However, the long-term benefits for Japanese

CI users have rarely been reported. In particular, language development after CI among Japanese children has not often been investigated. Language development outcomes among children with prelingual hearing impairment have been studied in Indo-European languages, but language differences may have an effect on language development in children with CI. In addition, differences in national and local education systems may make a difference to language development. To determine the effect of CI, we examined language development in different language and/or social 
systems.

Language differences may add other difficulties; for example, interpretation of Japanese language test results may not be comparable with that of English or other European language tests. To reduce these difficulties, we have established the assessment package of the language development for Japanese hearing-impaired children (ALADJIN) as a language performance evaluation tool for hearing-impaired children. ALADJIN includes several Japanese language tests that are directly comparable with previously reported English tests, including the Peabody Picture Vocabulary Test-Revised (PVT-R) and Test for Reception of Grammar (TROG)-like syntax tests (e.g., the Syntactic processing Test for Aphasia; STA). These tests all have their own distinctive emphasis and evaluate different aspects or domains of language.

In 2010, we assessed the current status of hearing-impaired children in Japan through a project called Research on Sensory and Communicative Disorders (RSCD). ALADJIN was used in this nationwide research project. The RSCD was originally intended to assess the effectiveness of interventional methods for hearing-impaired children. As part of the RSCD 
survey, we evaluated the domain-specific language status of Japanese hearing-impaired children with CI, not only in selected institutes and schools that potentially yield biases, but in a wide variety of institutes in Japan.

Thus, the objective of this study was to evaluate the development of interpersonal communication skills (IPCS) in hearing-impaired children with CI using the ALADJIN data set from the RSCD nationwide research project.

\section{Materials and Methods}

All ALADJIN tests were conducted by trained audiologists, speech pathologists, or deaf school teachers in a noise-minimized compartment. Audiometry for evaluation of hearing level, pure-tone threshold, speech discrimination test, and speech intelligibility rating (5) were measured in a sound-attenuated room of the relevant hospital. The study design was approved by the ethics review board of the Association of Technical Aids.

\section{Subjects}


In 2009, 124 institutes were participated in the RSCD project and 638 hearing-impaired children were registered; written informed consent was obtained from their parents. Open recruitment was conducted not only in institutes for hearing-impaired children, i.e., deaf schools and hard of hearing schools, but also in mainstream schools, day-care nurseries, and hospital/clinic training programs.

Most children included in this project were within the age range from 4 years ( 2 years before elementary school entrance; -2 grade) to 12 years $\left(6^{\text {th }}\right.$ grade of elementary school; +6 grade $)$ and confirmed to have congenital hearing impairment (average hearing level $>70 \mathrm{~dB}$ at 4 years of age). Children who were discernibly unable to complete the ALADJIN tests due to additional handicaps were excluded. 282 (44.2\%) participating children were CI users, and about $45 \%$ of the hearing-impaired children of each age group were CI users (Figure 1). Subjects were classified into four groups as follows: 1) "unil CI-only" group with unilateral CI users, 2) "CI-HA" group with CI plus conventional HA users (also called the bimodal stimulation group), 3) "bil-CI" group with bilateral CI users, and 4) "HA-only" group with HA users. The number of CI children in each age 
group is given in Figure 2. 84 children (35 males and 49 females, 29.8\%) in the unil CI-only group were diagnosed as hearing-impaired at 12.5 months on average. In the CI-HA group, 196 children (99 males and 97 females, $69.5 \%$ ) were diagnosed as hearing-impaired at 10.9 months on average. Two children ( 1 male and 1 female, $0.7 \%$ ) were bilateral CI users (bil-CI group). In the HA-only group, 356 children were diagnosed as hearing-impaired at 13.3 months and fitted at first hearing aids at 17.2 months ( 0 to 74 months) on average. Age at first fitting hearing aids in the children with CI was 15 months (2 to 47 months).

In order to reduce the influence of developmental disabilities in our evaluation of the language tests (ALADJIN), participating children with birth weights $<1800 \mathrm{~g}$, PARS scores $>11$ points, and RCPM scores $<2$ SD of the average were excluded. The numbers of subjects in each group were evaluated in the language tests as follows: 60 unil CI-only users, 128 CI-HA users, and 203 HA-only users. No significant differences in the scores of PARS and RCPM among the unil CI-only, CI-HA, and HA-only users were found (Figure 3). 


\section{Test battery}

We used the test for question-answer interaction development (TQAID) as a tool to measure IPCS function objectively. To let children understand a content of task, their favorite mode of communication (aural, sign language, total communication) were used to perform the language tests. $80 \%$ of subjects used aural communication as major mode in the domestic life. The following tests were also used to evaluate IPCS the day after administration of the TQAID.

The Word Fluency Test (WFT) was conducted as a measure of productive vocabulary $(6,7)$. Children were asked to produce as many words as possible from a certain category in 60 seconds. The words, represented either orally or manually, were carefully counted, excluding onomastic words. The Japanese version of the PVT-R (8) and the Standardized Comprehension Test for Abstract Words (SCTAW) (9) were also conducted to evaluate comprehensive vocabulary. An adjusted score was used in this study. The SCTAW consists of 32 or 45 abstract words selected from Japanese school textbooks. The details of how this method has been adapted for hearing-impaired children have been reported in 
previous studies $(9,10)$. Only school-aged children were subjected to this test.

The STA evaluates comprehension and production of syntactic structures. The children were asked to choose one of the four pictures appropriate to the tester's presentation (comprehension test) or to express a sentence according to a picture that the tester indicated (production test) (11). The tests evaluated understanding and expression of irreversible sentences, reversible sentences, Japanese suffixes (Jyo-Shi), and other syntactic structures, including relative pronouns.

To evaluate additional handicaps other than hearing impairment, the Pervasive Developmental Disorder ASJ Rating Scale (PARS) test for autistic tendency (12) and Raven's Colored Progressive Matrices (RCPM) test of non-verbal intelligence (13) were used only in school-aged children.

\section{Statistical analyses}

All statistical values were calculated using IBM SPSS Statistics 18 software (IBM Corp., Armonk, NY, USA). Correlations and standard deviations within each group were examined. The scores of the language 
tests (PARS, RCPM, PVT-R, SCTAW, WFT, STA, and TQAID) were translated as Z-scores from the results of each test in each age group.

\section{Results}

There were significant $(p<0.01)$ differences in the scores of average hearing loss level, average threshold level with hearing devices, maximum speech discrimination score, and speech intelligibility rating between CI users (unil CI-only or CI-HA users) and HA-only users (Figure 4). Hearing loss level of CI users was significantly lower than that of HA-only users. However, the threshold level, maximum speech discrimination scores, and speech intelligibility rating of CI users were significantly better than those of HA-only users. The scores of the PVT-R, SCTAW, and WFT tests, which evaluate vocabulary, were higher in CI users than in HA-only users (Figure 5). There was a significant difference $(p<0.01)$ in the results of the PVT-R test. The scores of the STA (Figure 6) and TQAID (Figure 7) in CI-HA users were significantly higher $(\mathrm{p}<0.05)$ than those in the unil CI-only group.

The high correlation $(\mathrm{r}=0.52)$ has been found between the age of CI 
and maximum speech discrimination score (Figure 8). The average scores of speech and language tests in the implanted children before 24 months of age have been better than those in the implanted children after 24 months of age (Table 1). The average scores of WFT (evaluation of productive vocabulary) and comprehension and production tests of STA (evaluation of syntactic structure) were significantly better in the implanted children before age of 24 months compared with the implanted children after age of 24 months.

\section{Discussion}

To evaluate the language development in the typical hearing-impaired children, we have made exclusionary criteria to standardize the subjects in this study. We excluded the hearing-impaired children with birth weights $<1800 \mathrm{~g}$ who scored $>11$ points on the PARS test and $<2$ SD on the RCPM. Very low birth weight children are at a high risk of neurosensory disability, including developmental delay, behavioral problems, and learning disabilities (14). Long-term follow-up studies have also emphasized the prevalence of significant neuropsychological and 
behavioral deficits at school age in children of very low birth weight (15). Therefore, we excluded children with birth weights $<1800 \mathrm{~g}$ to reduce the influence of developmental disabilities in our evaluation of communication skills. The PARS and RCPM tests determine the presence of pervasive developmental disorders and non-verbal intelligence, respectively. The scores in these tests were not significantly different among unil CI-only, CI-HA, and HA-only users. Consequently, children with developmental disabilities were excluded from the present study. However, children with ANSD (auditory neuropathy spectrum disorder) could not been excluded, because we did not get the data of ABR and OAE in this study.

Speech development for prelingual deaf children depends on optimal amplification with a CI or HA. Language acquisition is a high priority among deaf children who receive CI. During the 1990s, the following factors were considered to be associated with good speech development: age at implantation, duration of deafness, amount of daily use, mode of communication, and absence of other handicaps. Dettman et al. (16) reported that infants with implantation during the first year of life had significantly faster rates of receptive and expressive language 
development than those with implantation in the second year of life. On the other hand, another study found no significant differences in the performance in terms of spoken word recognition and expressive language development between children with implantation in the first and second years of life (17). In our study, early CI was more effective for better speech discrimination and children with CI before the second year of life had significantly better scores of productive vocabulary and comprehensive and productive syntax.

Early intervention has a strong influence on language outcomes in most, but not all, hearing-impaired children. The degree of hearing loss is an important factor in the modeling of speech production and spoken language outcomes. Several studies have demonstrated a clear relationship between the degree of hearing loss and language outcome (18). In our study, the average age at diagnosis of hearing loss in children with CI was 11.4 months. Age at diagnosis in CI-HA users (10.9 months) was earlier than in unil CI-only users (12.5 months) and HA-only users (13.3 months). Better speech and language development was found in CI-HA users compared with unil CI-only users. 
The degree of hearing loss in CI users was higher than in HA-only users. Speech discrimination score and intelligibility rating were higher in CI users than in HA-only users. The degree of hearing loss was significantly negatively correlated with speech discrimination and intelligibility. However, no clear relationship between the degree of threshold with the amplification devices and speech discrimination and intelligibility was found. The degree of threshold with amplification is thus a predictive factor of speech discrimination and intelligibility. It is beneficial for the CI to establish the better threshold level because fitting method is completely different. This study confirmed that CI has a positive influence on speech discrimination and intelligibility in severely hearing-impaired children. However, 124 institutions were participated in this study as nationwide research project, so there might be a confounding variable for selection of amplification devices (CI/HA vs $\mathrm{CI} / \mathrm{CI}$ vs uni $\mathrm{CI})$.

In evaluating auditory performance, formal speech perception tests, such as open-set and closed-set tests, are often used in children with CI. Communication skills, including auditory, speech, and language development for congenital and prelingual deaf children with CI, are 
influenced by a wide variety of factors. Several studies have reported that factors such as gender, nonverbal intelligence, estimated family income, communication mode, performance IQ, working memory capacity, articulation rate, and verbal rehearsal speed may predispose a child to better or poorer outcomes with a CI $(3,17)$.

We developed ALADJIN as a set of language tests to evaluate IPCS ability. Results of this assessment showed that CI was more effective for the development of comprehensive and productive vocabulary compared with HA, and bimodal hearing with CI and HA positively influence the development of vocabulary (comprehensive and productive), syntax (comprehensive and productive), and IPCS compared with unilateral hearing with CI. Consequently, we can conclude that early CI, especially in combination with HA, is useful in the development of communication skills.

\section{Acknowledgment}

We thank all participants of this study, including children, parents, and staff of the Research on Sensory and Communicative Disorders (RSCD) project 
who collaborated with the Association for Technical Aids (Tokyo, Japan).

This work was supported by the Division of Hearing Impairment, RSCD of the Ministry of Health, Labour and Welfare, Tokyo, Japan.

\section{References}

1. Kirk KI, Miyamoto RT, Lento CL, Ying E, O’Neill T. Effects of age at implantation in young children. Ann Otol Rhinol Laryngol Suppl 2002;189:69-73.

2. Zimmerman-Phillips S, Robbins AM, Osberger MJ. Assessing cochlear implant benefit in very young children. Ann Otol Rhinol Laryngol Suppl $2000 ; 185: 42-43$.

3. Moon IJ, Kim EY, Chu H, Chung WH, Cho YS, Hong SH. A new measurement tool for speech development based on Ling's stages of speech acquisition in pediatric cochlear implant recipients. Int J Pediatr Otorhinolaryngol 2011;75:495-499.

4. Geers AG, Nicholas JG, Moog JS. Estimating the influence of cochlear implantation on language development in children. Audiol Med 2007;5:262-273. 
5. Allen C, Nikolopoulos TP, Dyar D, et al: Reliability of a rating scale for measuring speech intelligibility after oediatric cochlear implantation. Otol Neurotol 2001:22:631-633

6. Riva D, Nichelli F, Devoti M. Developmental aspects of verbal fluency and confrontation naming in children. Brain and Language 2000;71:267-284.

7. Arffa S. The relationship of intelligence to executive function and non-executive function measures in a sample of average, above average, and gifted youth. Arch Clin Neuropsychol 2007;22:969-978.

8. Ueno K, Nagoshi S, Konuki S. Picture Vocabulary Test-Revised. Hiroshima, Japan: SACCESS Bell Co., Ltd. 2008 (in Japanese).

9. Haruhara N, Kaneko M, Uno A. The Standardized comprehension test of abstract words. Tokyo: Interuna Publishers, Inc. 2002 (in Japanese).

10. Kunisue K, Fukushima K, Kawasaki A, Maeda Y, Nagayasu R, Kataoka Y, et al. Comprehension of abstract words among hearing impaired children. Int J Pediatr Otorhinolaryngol 2007;71:1671-1679.

11. Nakajima R, Horai T, Sugata T, Tatsumi H, Hamanaka T. Syntactic and Communication Abilities of Aphasics As Assessed by CADL,STA and 
CADL Family Questionnaire. Jpn J Logopedics Phoniatrics 1997;38:161-168.

12. Yamada A, Suzuki M, Kato M, Suzuki M, Tanaka S, Shindo T, et al. Emotional distress and its correlates among parents of children with pervasive developmental disorders. Psychiatry Clin Neurosci 2007;61:651-657.

13. Weichbold V, Herka H. Performance of hearing impaired children on Raven's Coloured Progressive Matrices Test. Int J Pediatr Otorhinolaryngol 2003;67:1213-7.

14. Tamaru S, Kikuchi A, Takagi K, Wakamatsu M, Ono K, Horikoshi T, et al. Neurodevelopmental outcomes of very low birth weight and extremely low birth weight infants at 18 months of corrected age associated with prenatal risk factors. Early Hum Dev 2011;87:55-59.

15. Marlow N, Wolke D, Bracewell MA, Samara M. Neurologic and developmental disability at six years of age after extremely preterm birth. N Engl J Med 2005;352:9-19.

16. Dettman SJ, Pinder D, Briggs RJ, Dowell RC, Leigh JR. Communication development in children who receive the cochlear 
implant younger than 12 months: risks versus benefits. Ear Hear 2007;28 (2):11S-18S.

17. Holt RF, Svirsky MA. An exploratory look at pediatric cochlear implantation: is earliest always best? Ear Hear 2008;29:492-511.

18. Sininger YS, Grimes A, Christensen E. Auditory development in early amplified children: factors influencing auditory-based communication outcomes in children with hearing loss. Ear Hear 2010;31:166-185.

\section{Legends}

Figure 1 . The rate of CI-only users among the participating hearing-impaired children of each age group. About $45 \%$ of hearing-impaired children ( $>70 \mathrm{~dB}$ hearing level) in this study were CI-only users. CI: cochlear implant

Figure 2. Number of children in the CI-HA, unil CI-only, and bil-CI groups in each age range. CI plus HA users (bimodal stimulation) make up the majority of CI users. CI: cochlear implant, unil CI: unil CI-only users, bil-CI: bilateral CI users, CI-HA: HA and CI users 
Figure 3. The scores of PARS and RCPM tests in the HA-only, CI-HA, and unil CI-only groups. There were no significant differences in average scores among the groups. PARS: Pervasive Developmental Disorder ASJ Rating Scales for evaluating autistic tendency, RCPM: Raven's Colored Progressive Matrices test for evaluating non-verbal intelligence, CI: cochlear implant, HA: hearing aid

Figure 4. Hearing loss levels, threshold levels, maximum speech discrimination scores, and speech intelligibility scores in the HA-only, CI-HA, and unil CI-only groups. There are significant differences $(\mathrm{p}<$ 0.01) in hearing levels, threshold levels, speech discrimination scores, and intelligibility scores between the CI group (CI-HA or unil CI-only groups) and HA-only group. Children with CI achieve better threshold levels, speech discrimination, and intelligibility compared with HA-only users. ** $\mathrm{p}<0.01, \mathrm{CI}$ : cochlear implant, HA: hearing aid 
Figure 5. Scores of the PVT-R, SCTAW, and WFT tests in the HA-only, CI-HA, and unil CI-only groups. Scores of the PVT-R, SCTAW, and WFT tests in the CI-HA and unil CI-only groups were better than those in the HA-only group. A significant difference $(\mathrm{p}<0.01)$ was found in the scores of the PVT-R test. $* * \mathrm{p}<0.01$, PVT-R: Peabody Picture Vocabulary Test-Revised. SCTAW: Screening Test for Abstract Words. WFT: Word Fluency Test. Values in the longitudinal line indicate Z-score.

Figure 6. Scores of the STA test (comprehension and production) in the HA-only, CI-HA, and unil CI-only groups. STA test scores (comprehension) in the CI-HA group were significantly higher $(\mathrm{p}<0.05)$ than in the HA-only group. STA test scores (production) in the CI-HA group were significantly higher $(\mathrm{p}<0.05)$ than those in the unil CI-only and HA-only groups. ${ }^{*} \mathrm{p}<0.05$. STA: Syntactic Processing Test for Aphasia test. Values in the longitudinal line indicate Z-score.

Figure 7. The scores of TQAID test in the HA-only, CI-HA, and unil CI-only groups. 
The score of TQAID test in the CI-HA group is significantly $(\mathrm{p}<0.05)$ better than that in the unil CI-only group.

$* \mathrm{p}<0.05$, TQAID: test for question-answer interaction development is for evaluating the IPCS (interpersonal communication skills) function. Values in the longitudinal line indicate Z-score.

Figure 8 . The correlation between the age of cochlear implantation and maximum speech discrimination score The high correlation $(\mathrm{r}=0.52)$ has been found between the age of $\mathrm{CI}$ and maximum speech discrimination score.

Table 1. Average scores of language tests (ALADJIN) in children with CI before and after age of 24 months 
$\square \mathrm{Cl}$ घnon-Cl

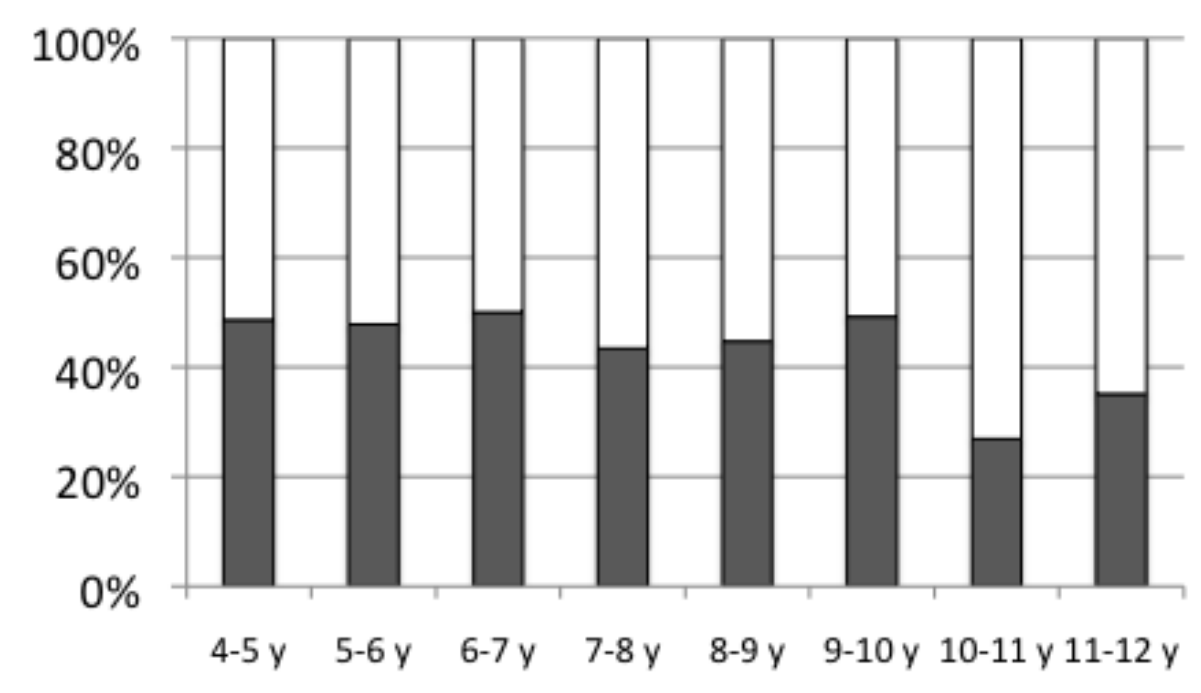

Figure 1 


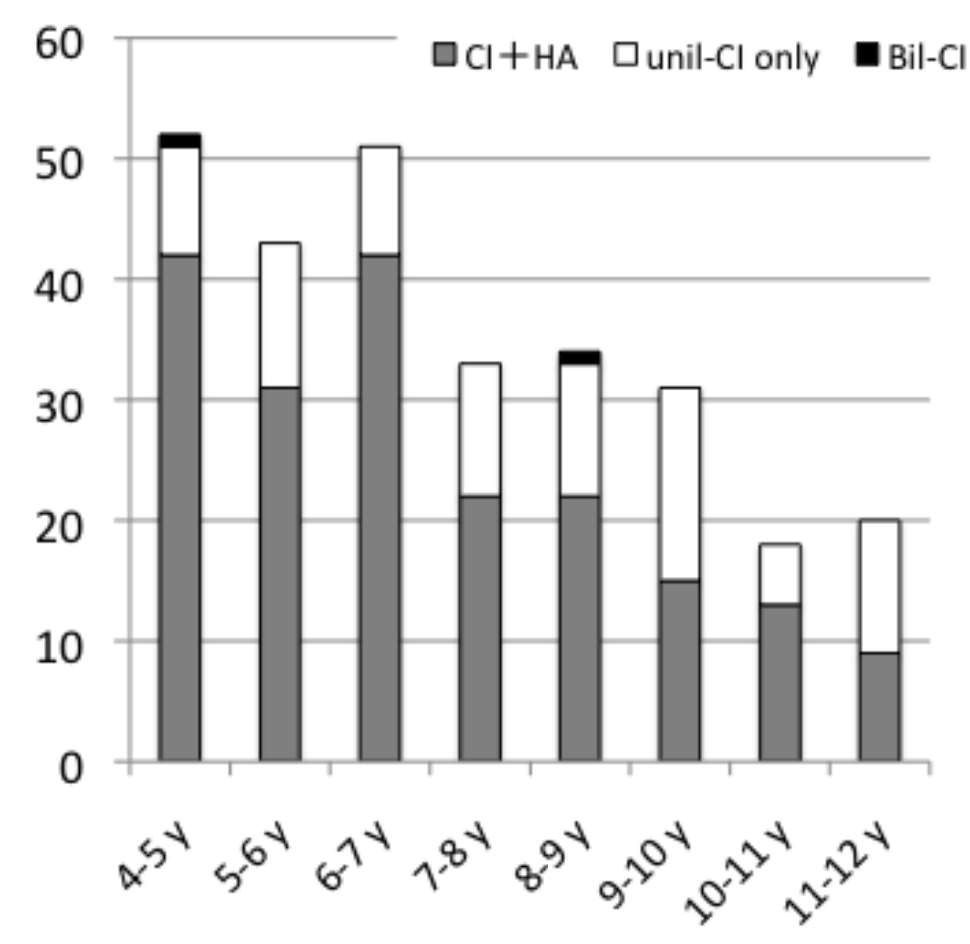

Figure 2 

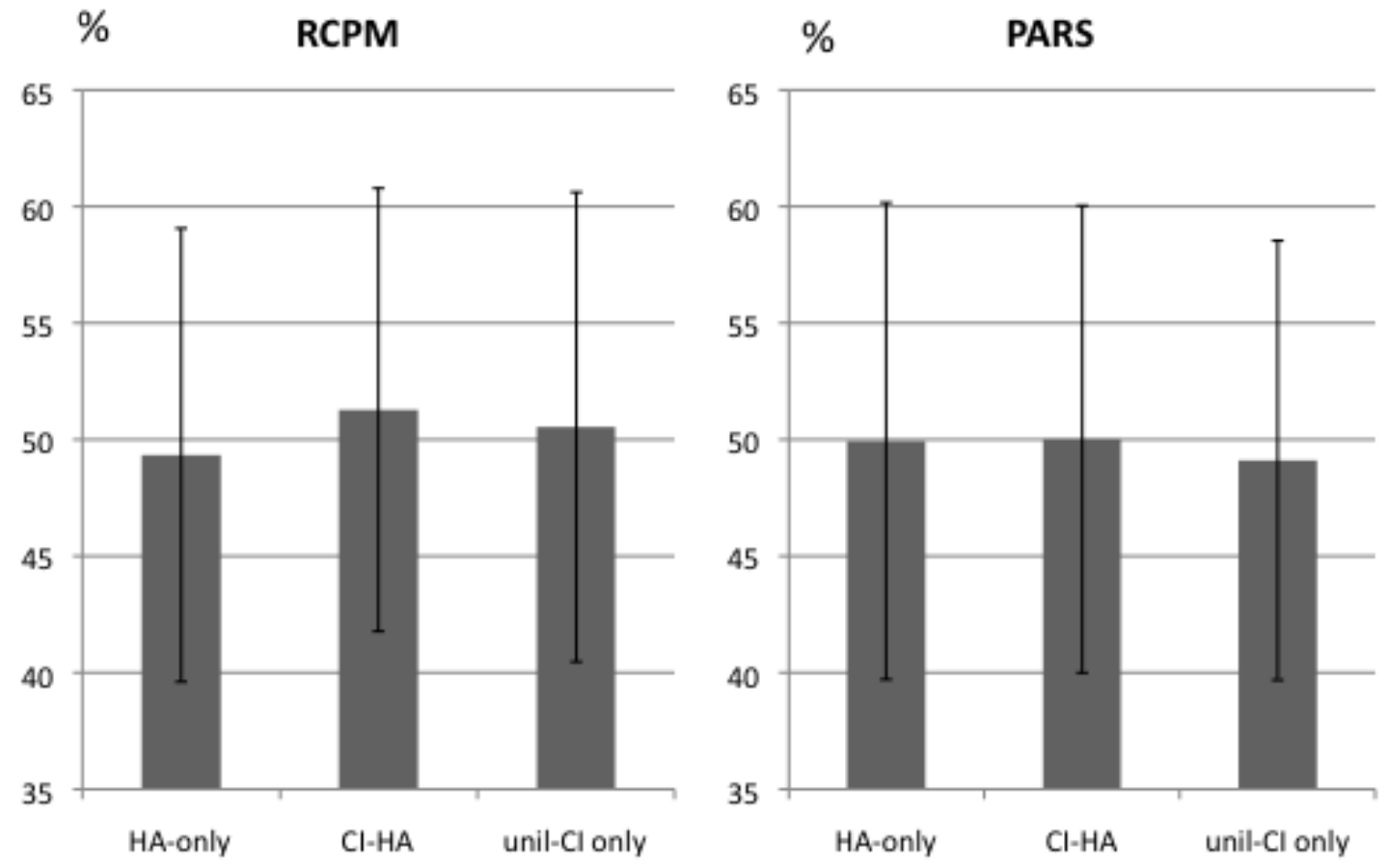

Figure 3 

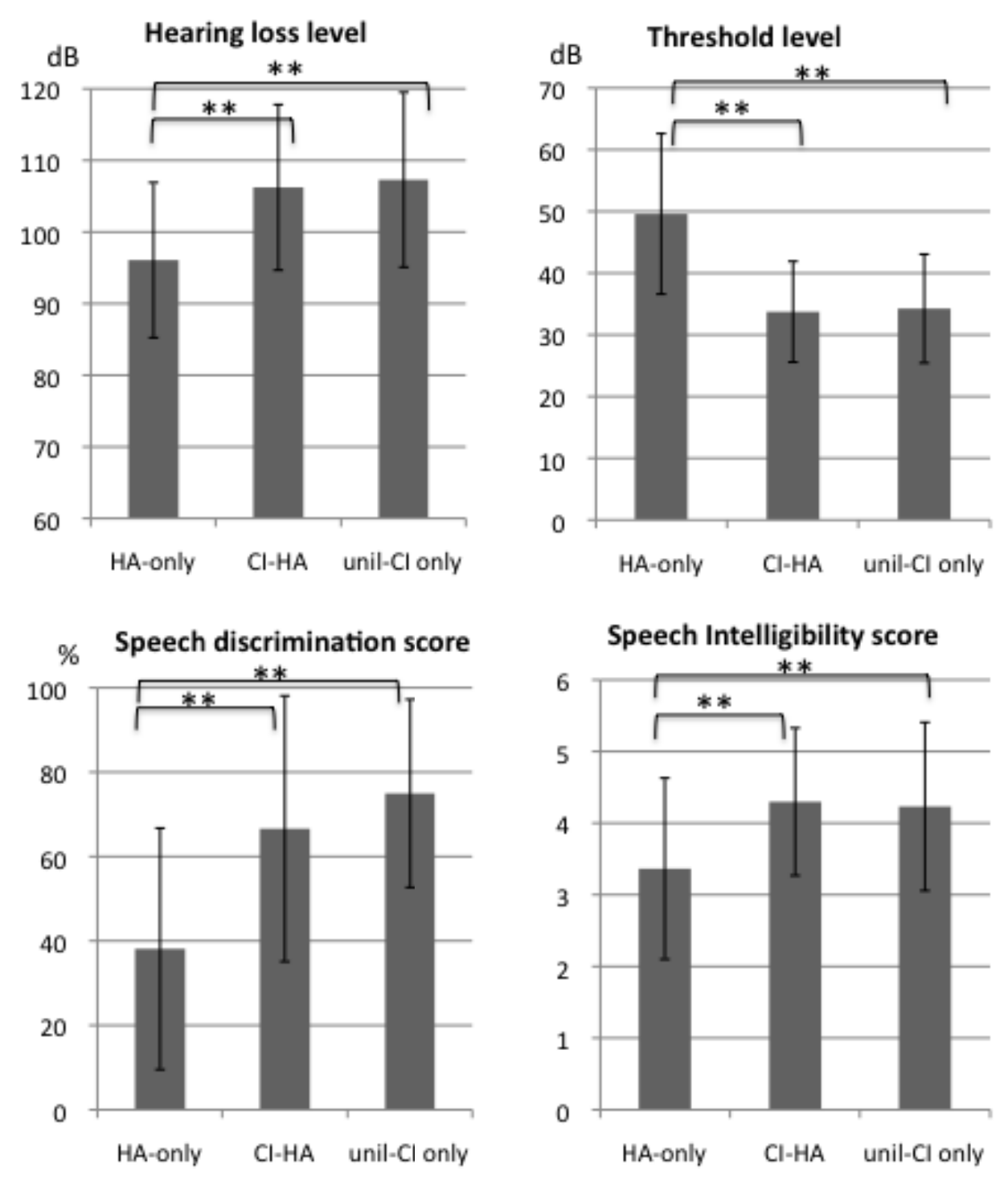

Figure 4 


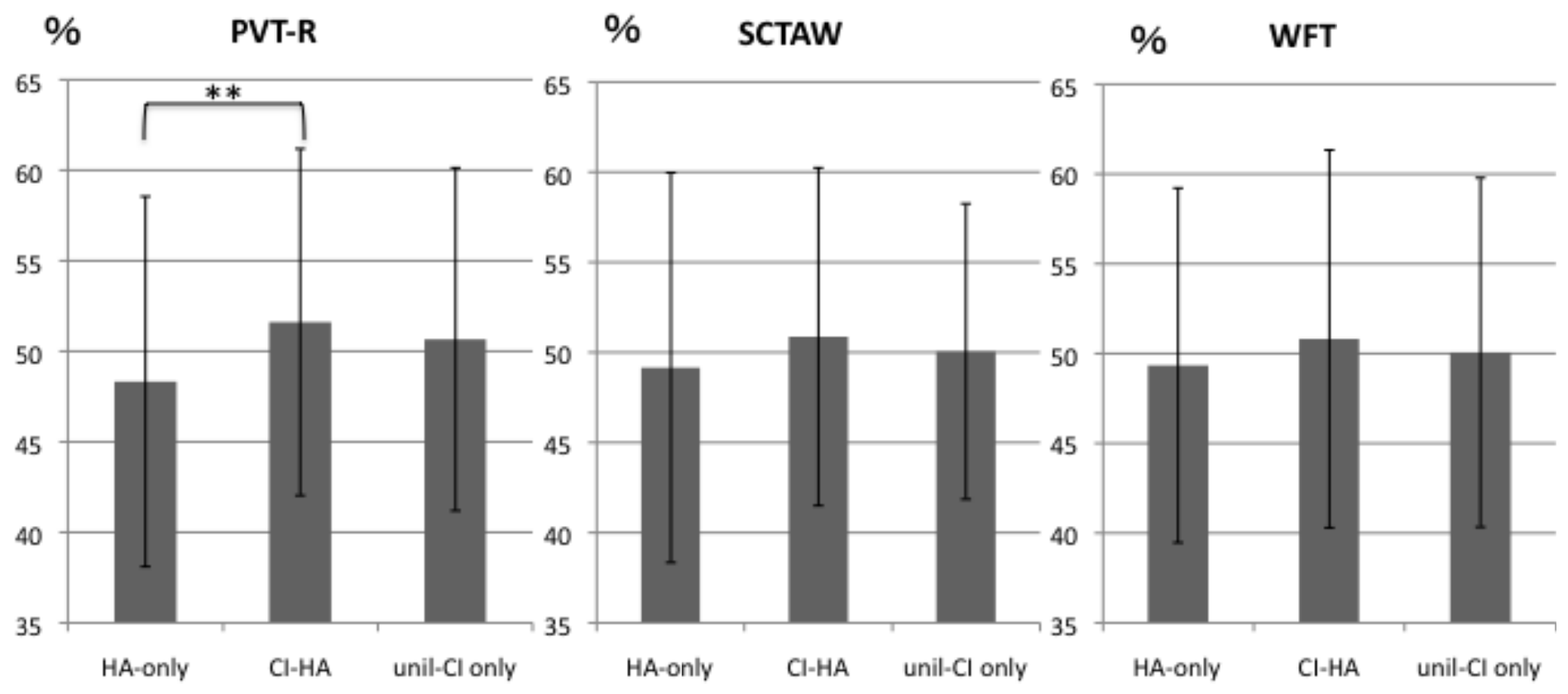

Figure 5 
$\% \quad$ STA (comprehension)

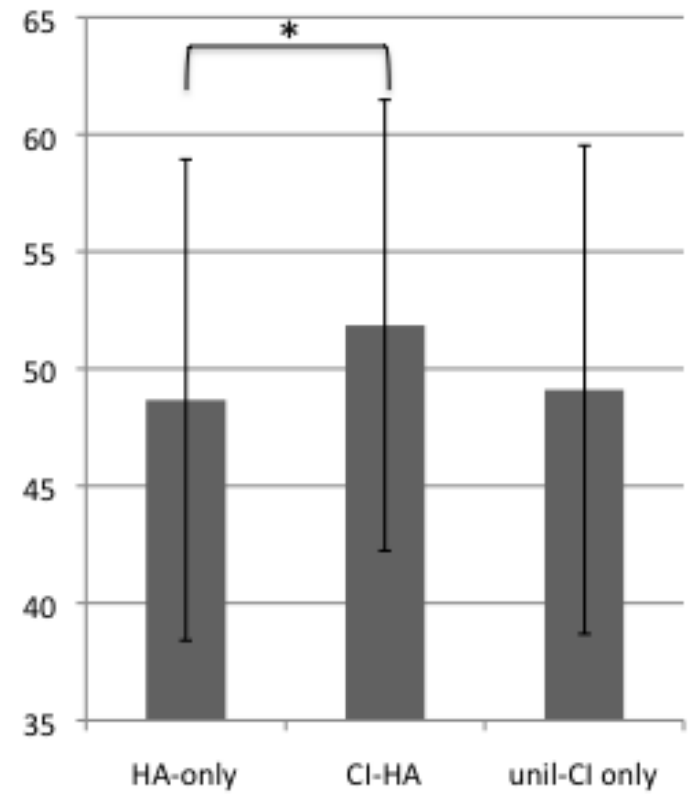

$\%$ STA (production)

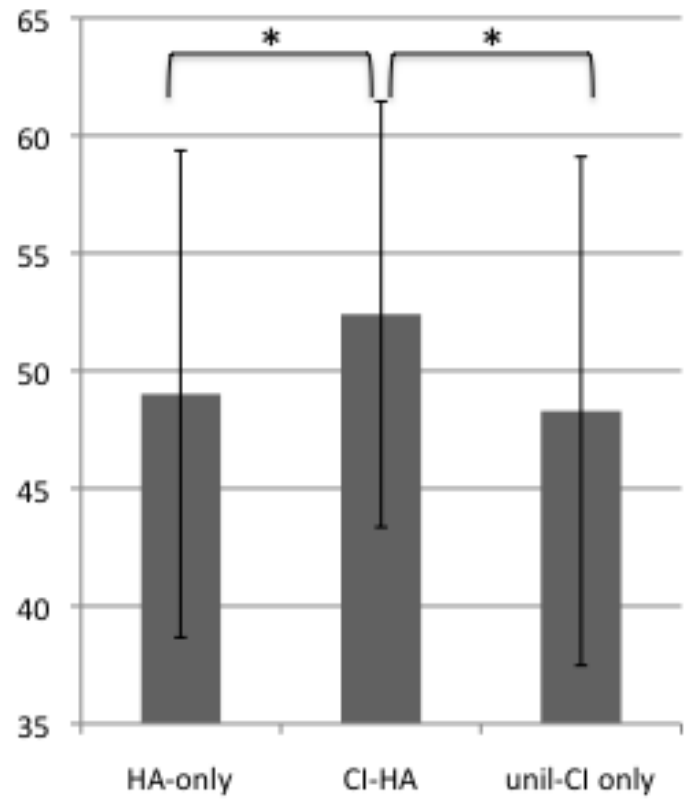


TQAID

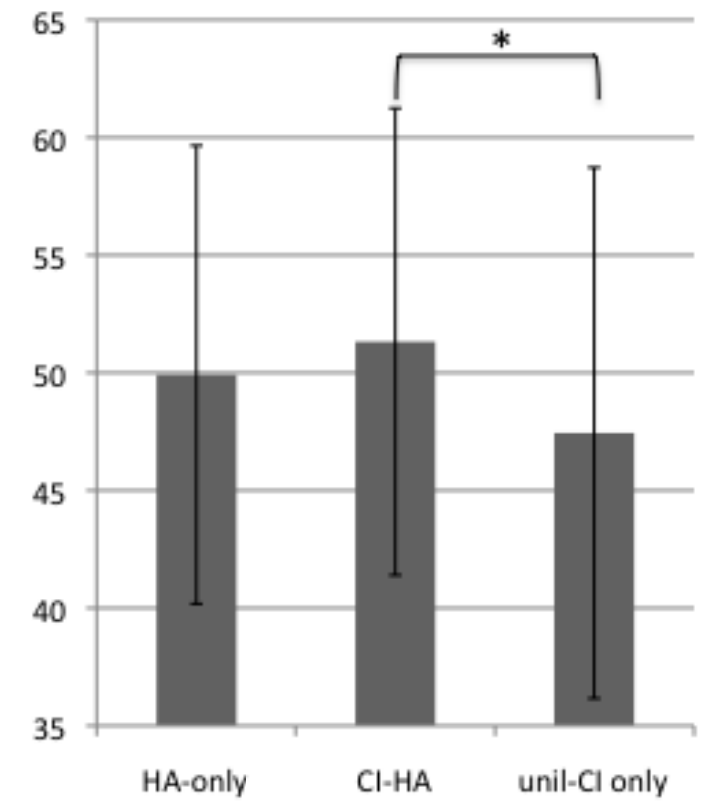

Figure 7 


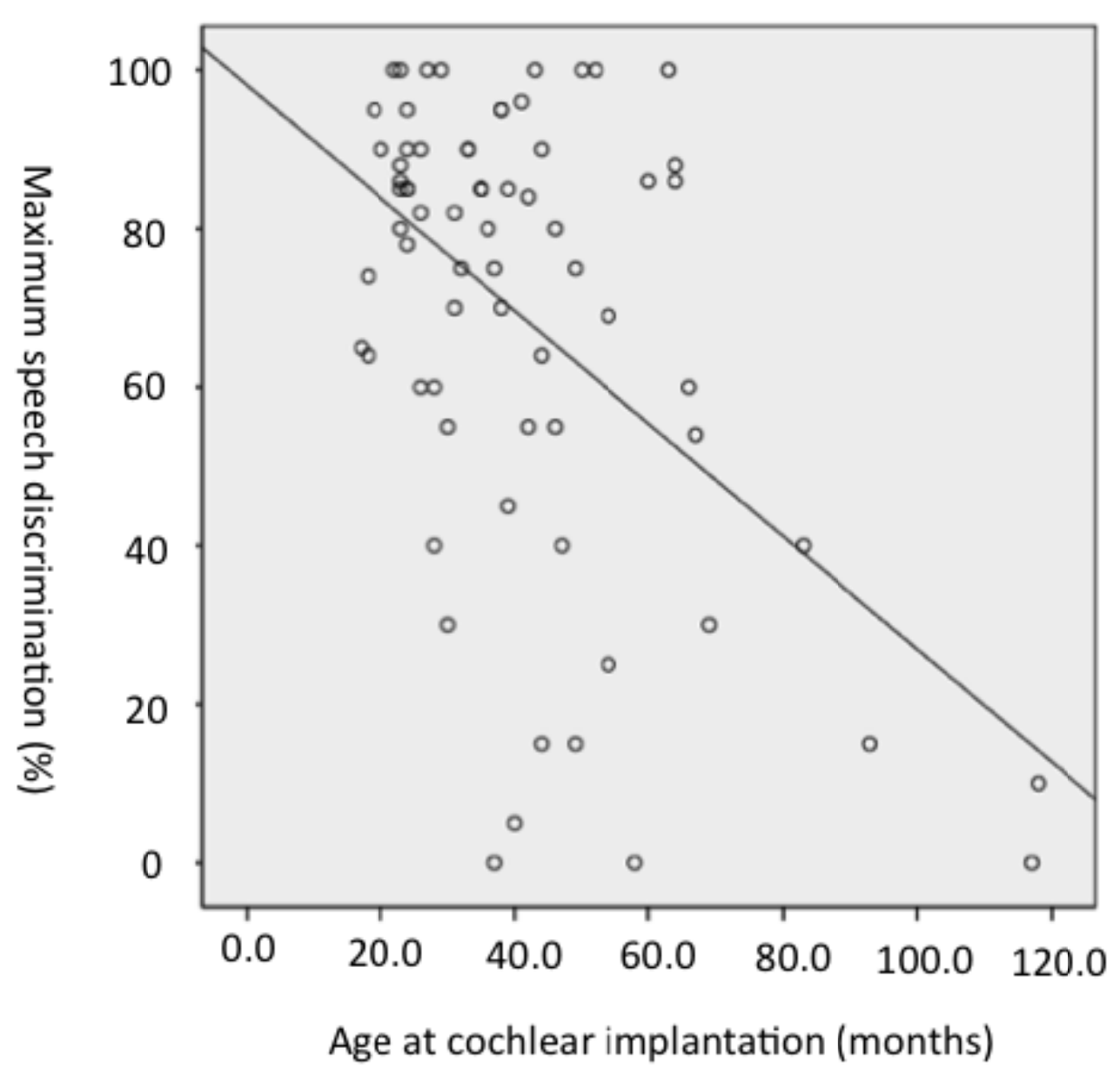

Figure 8 
Table 1 Average scores of language tests (ALADJIN) in children with CI before and after age of 24 months

\begin{tabular}{|l|c|c|c|c|c|c|c|c|}
\hline & PVT-R & WFT & SCTAW & STA (Com) & STA (Pro) & TQAID & RCPM & PARS \\
\hline CI after $24 \mathrm{mo}(\mathrm{N}=29)$ & 29.5 & 12.9 & 13.7 & 23.1 & 34.1 & 209.4 & 27.5 & 5.3 \\
\hline CI before $24 \mathrm{mo}(\mathrm{N}=16$ & 32.2 & 15.6 & 13.8 & 26.2 & 39.0 & 229.7 & 28.1 & 4.5 \\
\hline $\mathrm{t}-$-value & 0.19 & 0.02 & 0.99 & 0.04 & 0.04 & 0.06 & 0.77 & 0.30 \\
\hline
\end{tabular}

PVT-R: Peabody Picture Vocabulary Test-Revised, WFT: Word Fluency Test, SCTAW: Standardized Comprehension Test for Abstract Words

STA (Com): Syntactic processing Test for Aphasia (Comprehension), STA (Pro): Syntactic processing Test for Aphasia (Production)

TQAID: Test for Question-Answer Interaction Development, RCPM: Raven's Colored Progressive Matrices,

PARS: Pervasive Developmental Disorder ASJ Rating Scale, CI: Cochlear Implantation, mo: months, N: Number 